\title{
Design of Miniaturized Multiband Filters Using Zero Order Resonators for WLAN Applications
}

\author{
Maryam Shafiee, ${ }^{1}$ Mohammad Amin Chaychi Zadeh, ${ }^{2}$ and Homayoon Oraizi ${ }^{2}$ \\ ${ }^{1}$ Department of Electrical Engineering, Arizona State University, Tempe, AZ 85287, USA \\ ${ }^{2}$ Department of Electrical Engineering, Iran University of Science and Technology, Narmak, Tehran 16846 13114, Iran \\ Correspondence should be addressed to Maryam Shafiee; mshafiei@asu.edu
}

Received 23 November 2014; Revised 15 February 2015; Accepted 18 February 2015

Academic Editor: Giancarlo Bartolucci

Copyright (c) 2015 Maryam Shafiee et al. This is an open access article distributed under the Creative Commons Attribution License, which permits unrestricted use, distribution, and reproduction in any medium, provided the original work is properly cited.

\begin{abstract}
The objective of this paper is to design miniaturized narrow- and dual-band filters for WLAN application using zero order resonators by the method of least squares. The miniaturization of the narrow-band filter is up to $70 \%$ and that of the dual-band filter is up to $64 \%$ compared to the available models in the literature. Two prototype models of the narrow-band and dual-band filters are fabricated and measured, which verify the proposed structure for the filter and its design by the presented method, using an equivalent circuit model.
\end{abstract}

\section{Introduction}

Mobility of components and equipment is a requirement in mobile communication systems. Therefore, considerable effort has gone into the miniaturization of devices. Furthermore, various techniques of multibanding have been devised for various functions. Particularly, different techniques have been proposed to make components dual-band, which are considered conventional methods, such as series connection of two separate band pass filters, step impedance resonators, and defected ground structures. All of these methods have some limitations on the reduction of device dimensions, because they are based on half-wave resonators $[1,2]$.

Recently, metamaterials have found wide applications, such as miniaturization of microwave devices, due to their unique properties and nonlinear dispersion curves. Zero order resonance (ZOR) metamaterials have the distinct property of infinite wavelength, which makes the resonance frequency independent of their dimensions. Consequently, the ZOR component may be fabricated as small as specified to realize the desired circuit elements for the required resonance frequency [3]. This unique characteristic of ZORs has been used in microwave components, such as filters [4-6].

In this paper, we use the method of least mean square error (LMS) as a contribution to design and fabricate a narrow-band metamaterial filter and also a dual-band filter for application in WLAN systems. The main advantage and feature of the proposed filters are their compactness and miniaturization, which is up to $70 \%$ smaller than conventional components in the literature [7].

\section{Design Procedure}

The proposed zero order resonance (ZOR) cell is depicted in Figure 1. The two interdigital capacitors provide the left-handed capacitance and right-handed inductance. The meander inductance and T-junction are inserted in the circuit to control and adjust the right-handed section of the device. Its equivalent circuit is shown in Figure 2. The values of capacitors and inductors are extracted from the formulas in the literature [8-11].

The error function is explicitly expressed as the function of geometrical dimensions of various sections of the filter. In other words, the lumped elements ( $L$ and $C$ ) of the equivalent circuit of the filter in Figure 2 are expressed in terms of the geometrical dimensions of the filter structure, which are given in literature. The overall transmission matrix of the equivalent circuit is first derived, which is then converted to the scattering parameters. Thus, the scattering parameters of equivalent circuit might be obtained by the available relations of the T-matrix in terms of its physical dimensions [12]. 


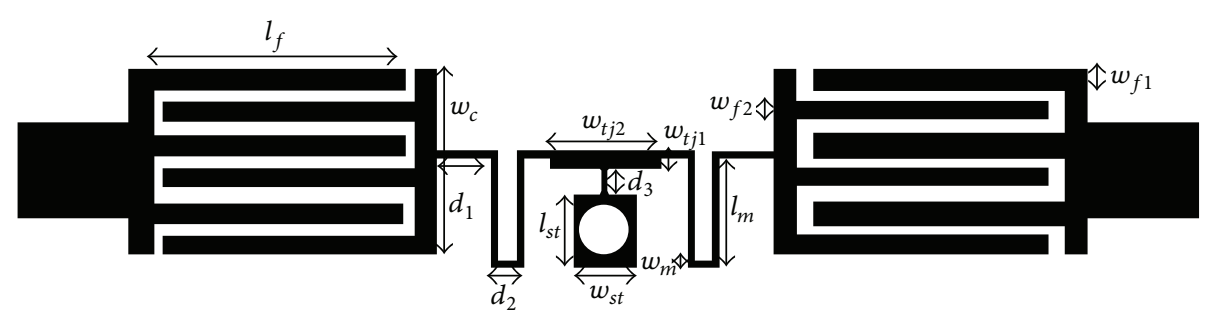

FIGURE 1: The microstrip layout of the metamaterial unit cell: $w_{m}=0.1 \mathrm{~mm}, l_{f}=4.55 \mathrm{~mm}, w_{f 1}=0.3 \mathrm{~mm}, w_{f 2}=0.4 \mathrm{~mm}, g a p_{1}=0.3 \mathrm{~mm}$, $\operatorname{gap}_{2}=0.2 \mathrm{~mm}, w_{s t}=1.14 \mathrm{~mm}, l_{s t}=1.3 \mathrm{~mm}, d_{1}=1 \mathrm{~mm}, d_{2}=0.45 \mathrm{~mm}, l_{m}=2.0 \mathrm{~mm}, w_{t j 1}=0.3 \mathrm{~mm}$, and $w_{t j 2}=0.1 \mathrm{~mm}$.

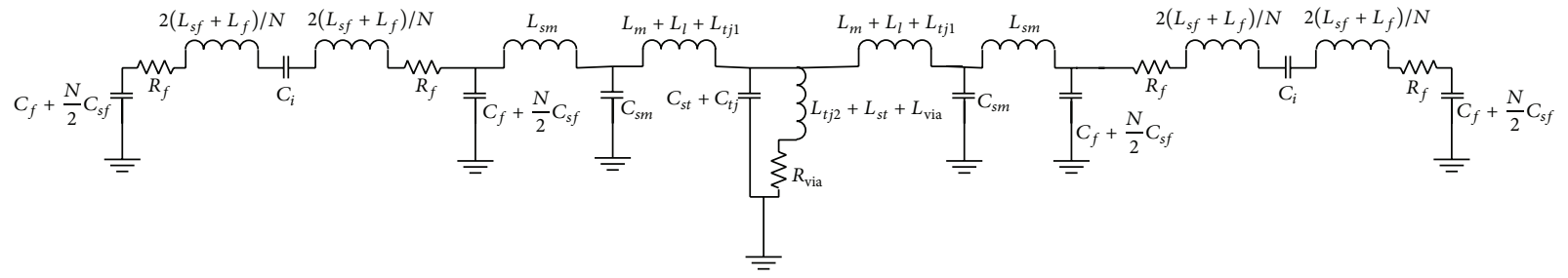

FIGURE 2: The proposed equivalent circuit of metamaterial unit cell.

We now refer to the specified frequency response of the bandpass filter, as shown in Figure 3. It is composed of the lower $\left(f_{L}\right.$ to $\left.f_{f}\right)$ and upper $\left(f_{l}\right.$ to $\left.f_{U}\right)$ stop bands, with attenuation $g_{s}$, the lower $\left(f_{f}\right.$ to $\left.f_{0}-\mathrm{BW} / 2\right)$ and upper $\left(f_{0}+\right.$ $\mathrm{BW} / 2$ to $\left.f_{l}\right)$ transition bands, with the attenuations $g_{t f}=$ $\left(g_{s} /\left(f_{f}-\left(f_{0}-\mathrm{BW} / 2\right)\right)\right)\left(f_{i}-f_{f}\right)$ and $g_{t s}=\left(g_{s} /\left(f_{f}-\left(f_{0}+\right.\right.\right.$ $\mathrm{BW} / 2)))\left(f_{i}-f_{l}\right)$, and the pass band with center frequency $f_{0}$ and bandwidth BW and insertion loss $g_{p}$. Each frequency interval is divided into $N_{i}$ discrete frequencies. The accuracy of the error function increases for larger number of discrete frequencies, but the CPU time of computations also increases. Therefore, a trade-off should be considered between accuracy and computing time. We then construct an error function as

$$
\begin{gathered}
\varepsilon_{s}=\sum_{i \in \text { stop }}^{N_{1}}\left(\left|s_{21}{ }^{i}\right|_{\mathrm{dB}}-g_{s}\right)^{2}, \\
\varepsilon_{p}=\sum_{i \in \text { pass }}^{N_{2}}\left(\left|s_{21}{ }^{i}\right|_{\mathrm{dB}}-g_{p}\right)^{2}, \\
\varepsilon_{t f}=\sum_{i \in \text { first.transition }}^{N_{3}}\left(\left|s_{21}{ }^{i}\right|_{\mathrm{dB}}-g_{t f}\right)^{2}, \\
\varepsilon_{t s}=\sum_{i \in \text { sec.transition }}^{N_{4}}\left(\left|s_{21}{ }^{i}\right|_{\mathrm{dB}}-g_{t s}\right)^{2} \\
\Longrightarrow \varepsilon_{\text {total }} \\
=w_{p} \times \varepsilon_{p}+w_{t} \times\left(\varepsilon_{t s}+\varepsilon_{t f}\right)+w_{s} \times \varepsilon_{s},
\end{gathered}
$$

where $\varepsilon_{s}$ is the error due to the lower and upper stop bands, $\varepsilon_{p}$ is the error related to the pass band, and $\varepsilon_{t f}$ and $\varepsilon_{t s}$ are the errors from the lower and upper transition bands, as depicted in the filter frequency response in Figure 3. The weighting

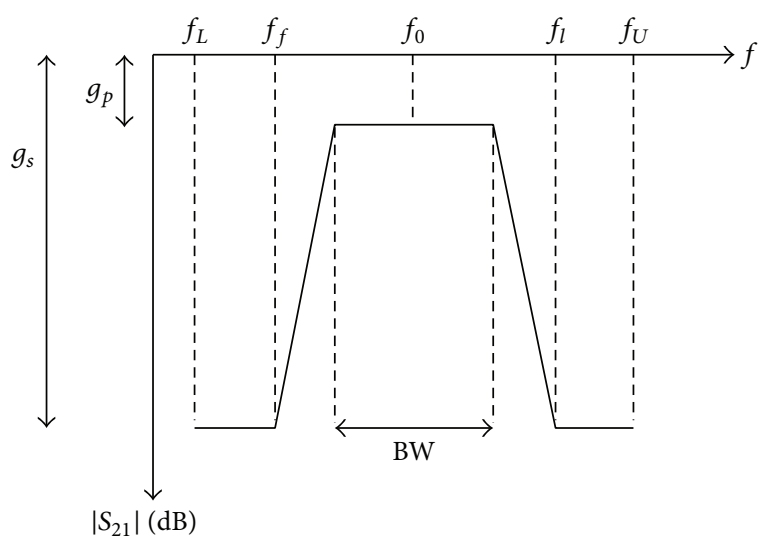

FIGURE 3: Design specifications of filter frequency response.

functions are $w_{s}, w_{p}$, and $w_{t}$ in order to adjust the value of each error term in the function of total error.

The error function can be constructed for multiband filters. The minimization of error function gives the optimum physical dimensions of the filter. The genetic algorithm (GA) as a global minimum seeking algorithm does not need initial values for variable, but it is quite slow. However, the conjugate gradient (CG) algorithm is a local minimum seeker algorithm, which needs initial values but it is quite fast. Therefore, a MATLAB code is written which combines GA and CG. First, GA is run to reach the vicinity of the absolute minimum point, but it is aborted prematurely and $\mathrm{GC}$ is activated to locate the minimum point quite fast. Since the design procedure based on the transmission line circuit model suffers some approximation and does not account for full wave performance of the filter, the CST Microwave Studio computer simulator is used to adjust the filter performance and obtain its optimum design [13]. 
TABLE 1: Line widths and lengths for the designed ZOR.

\begin{tabular}{|c|c|c|c|c|c|}
\hline \multicolumn{3}{|c|}{ Values before full wave optimization $(\mathrm{mm})$} & \multicolumn{3}{|c|}{ Values after full wave optimization (mm) } \\
\hline$w_{f}=0.47$ & $l_{s t}=0.90$ & $w_{t j 2}=0.10$ & $w_{f 1}=0.305$ & $w_{s t}=1.14$ & $w_{t j 2}=0.10$ \\
\hline gap $=0.23$ & $w_{m}=0.10$ & $l_{m}=2.00$ & $w_{f 2}=0.40$ & $l_{s t}=1.30$ & $l_{m}=2.00$ \\
\hline$l_{f}=4.95$ & $d_{1}=1.14$ & $d_{2}=0.67$ & $\operatorname{gap}_{1}=0.30$ & $w_{m}=0.10$ & $d_{2}=0.45$ \\
\hline$w_{s t}=2.0$ & $w_{t j 1}=0.12$ & $d_{3}=0.91$ & $\operatorname{gap}_{2}=0.20$ & $d_{1}=1.12$ & $d_{3}=0.50$ \\
\hline & & & $l_{f}=4.55$ & $w_{t j 1}=0.30$ & \\
\hline
\end{tabular}

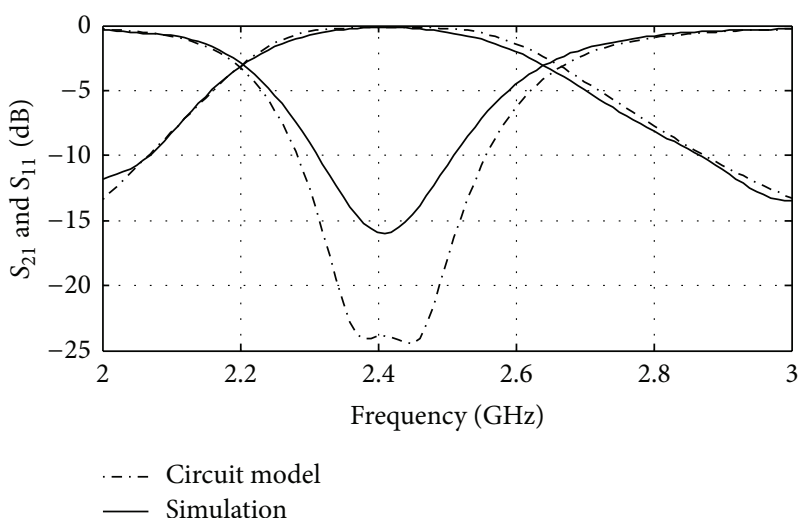

FIgURE 4: The frequency response of the ZOR unit cell obtained by the equivalent circuit and simulation software.

\section{Design of a ZOR Unit Cell}

We design a ZOR unit cell according to the following specifications specified in Figure 3, namely, centre frequency $f_{0}=2.4 \mathrm{GHz}, \mathrm{BW}=10 \%$, maximum insertion loss $g_{p}=$ $0.5 \mathrm{~dB}$, maximum stop band rejection $g_{s}=20 \mathrm{~dB}$ in the bands $1-2 \mathrm{GHz}$ and $3-4 \mathrm{GHz}, f_{f}=2.0 \mathrm{GHz}, f_{l}=3.0 \mathrm{GHz}, N_{1}=51$, $N_{2}=212$, and $N_{3}=N_{4}=45$. The minimization of error function determines the dimensions of the ZOR filter.

The frequency response of ZOR unit cell as obtained by the circuit model and full wave simulation using CST are shown in Figure 4. They are in a good agreement. In the circuit model of the unit cell, the interdigital sections of the filter are assumed identical, whereas in the full wave filter simulation they are assumed different by defining two separate variables for both gaps and widths to achieve the best possible result. Also, another optimization is performed on dimensions in full wave simulation as reported in Table 1.

The propagation constant of the structure could be computed from transmission parameters:

$$
\beta=-\varphi^{\text {unwrapped }}\left(S_{21}\right)+\zeta
$$

The unwrapped phase of $S_{21}$ is obtained by connecting the discontinuous sections of phase curves at $+\pi$ and $-\pi$, as shown in Figure 5. For the determination of the reference, where the electrical length $(\theta=\beta l)$ is zero, it is necessary to shift the unwrapped phase curve by $\zeta$ to remove the phase ambiguity [3]. Its value is obtained from the location of zero crossing point at the phase response curve.

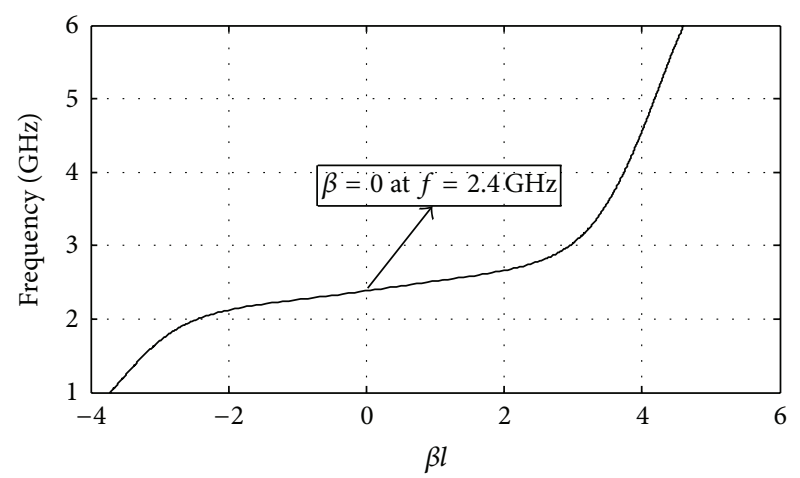

FIgure 5: The dispersion diagram of the ZOR unit cell.

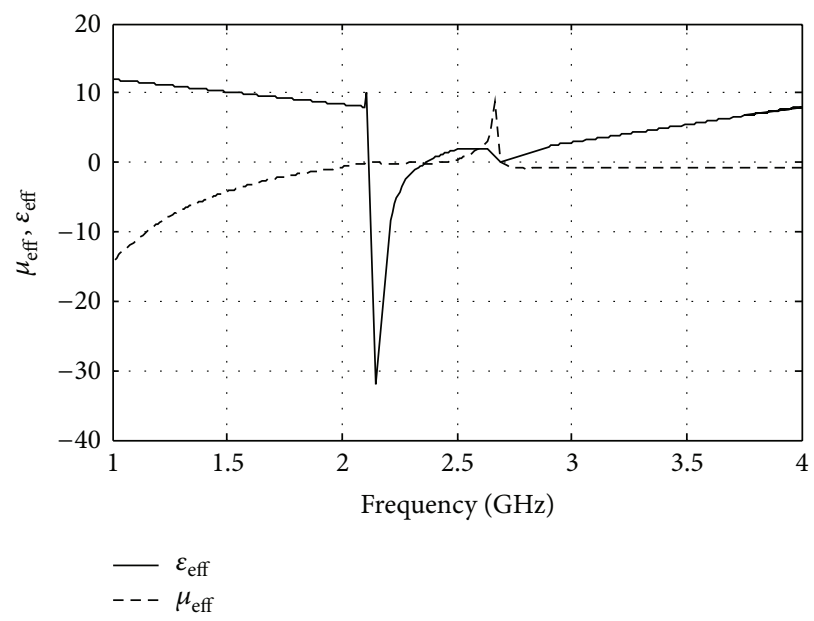

FIgURE 6: Constitutive parameters of the ZOR unit cell.

For the accurate characterization of metamaterial cell, its constitutive parameters are obtained by the procedure of effective media and are drawn in Figure 6 [14].

\section{Design of a Narrow-Band Filter}

In order to design a narrow-band filter with high rejection in the stop band, low insertion loss in the pass band, and sharp drop in the transition band, two ZOR unit cells are connected in series by an inductive coupling, as shown in Figure 7. The design procedure based on the LMS design method is similar to that of the unit cell. The scattering parameters are obtained 
TABLE 2: Dimensions of narrow-band filter designed by the proposed method.

(a) Dimensions of first unit cell

\begin{tabular}{|c|c|c|c|c|c|}
\hline \multicolumn{3}{|c|}{ Values before full wave optimization (mm) } & \multicolumn{3}{|c|}{ Values after full wave optimization (mm) } \\
\hline$w_{f}=0.3$ & $l_{s t}=1.00$ & $w_{t j 2}=0.10$ & $w_{f}=0.43$ & $l_{s t}=1.00$ & $w_{t j 2}=0.10$ \\
\hline gap $=0.588$ & $w_{m}=0.218$ & $l_{m}=2.65$ & gap $=0.56$ & $w_{m}=0.25$ & $l_{m}=2.70$ \\
\hline$l_{f}=4.226$ & $d_{1}=1.20$ & $d_{2}=0.50$ & $l_{f}=3.44$ & $d_{1}=1.2$ & $d_{2}=0.80$ \\
\hline$w_{s t}=1.17$ & $w_{t j 1}=0.20$ & $d_{3}=0.00$ & $w_{s t}=1.33$ & $w_{t j 1}=0.20$ & $d_{3}=0.00$ \\
\hline
\end{tabular}

(b) Dimensions of second unit cell

\begin{tabular}{|c|c|c|c|c|c|}
\hline \multicolumn{3}{|c|}{ Values before full wave optimization (mm) } & \multicolumn{3}{|c|}{ Values after full wave optimization (mm) } \\
\hline$w_{f}=0.31$ & $l_{s t}=1.05$ & $w_{t j 2}=0.10$ & $w_{f}=0.52$ & $l_{s t}=1.00$ & $w_{t j 2}=0.10$ \\
\hline gap $=0.69$ & $w_{m}=0.218$ & $l_{m}=2.65$ & gap $=0.7$ & $w_{m}=0.25$ & $l_{m}=2.70$ \\
\hline$l_{f}=3.59$ & $d_{1}=1.10$ & $d_{2}=0.70$ & $l_{f}=3.47$ & $d_{1}=0.92$ & $d_{2}=0.70$ \\
\hline$w_{s t}=1.33$ & $w_{t j 1}=0.20$ & $d_{3}=0.00$ & $w_{s t}=1.75$ & $w_{t j 1}=0.20$ & $d_{3}=0.00$ \\
\hline
\end{tabular}

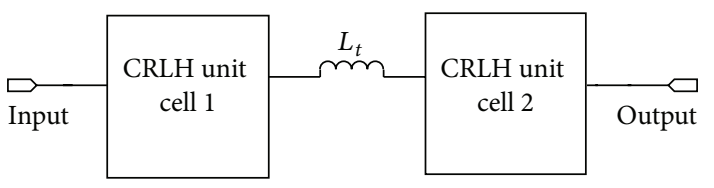

FIGURE 7: The equivalent circuit of the two metamaterial ZOR cells in the narrow-band and dual-band filters.

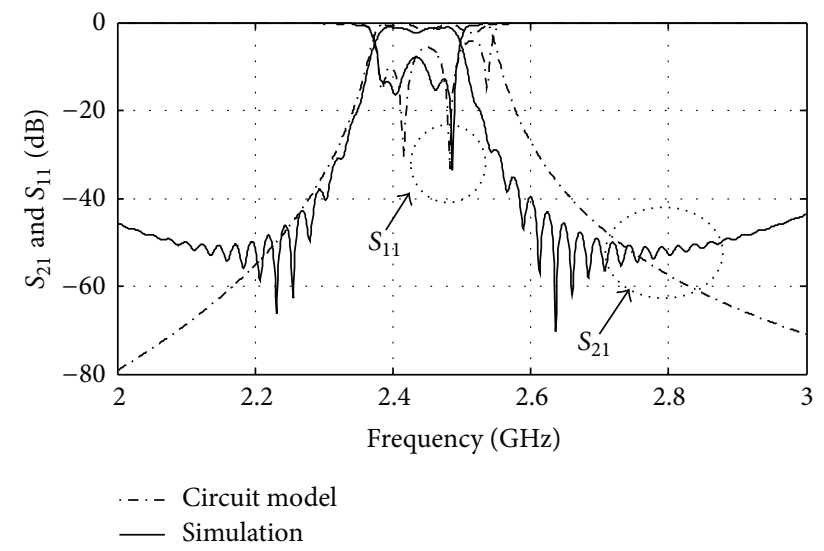

FIGURE 8: The frequency response of the narrow-band filter as $S_{21}$ obtained by simulations and circuit model.

from the transmission matrix, which are then used in the error function. The specifications of the narrow-band filter are $f_{0}=2.44 \mathrm{GHz}, \mathrm{BW}=100 \mathrm{MHz}, g_{p}=0.5 \mathrm{~dB}, g_{s}=30 \mathrm{~dB}$, $N_{1}=51, N_{2}=212$, and $N_{3}=N_{4}=45$.

The physical dimensions of the narrow-band filter obtained by the LMS design procedure and CST are given in Table 2.

The frequency responses of filter as the amplitudes of $S_{11}$ and $S_{21}$ obtained by the circuit model and computer simulation using CST Microwave Studio are illustrated in Figure 8. The full wave result shows a bandwidth of $80 \mathrm{MHz}$ from $2.36 \mathrm{GHz}$ to $2.48 \mathrm{GHz}$. The dispersion diagram of the proposed filter is depicted in Figure 9. The appearance of

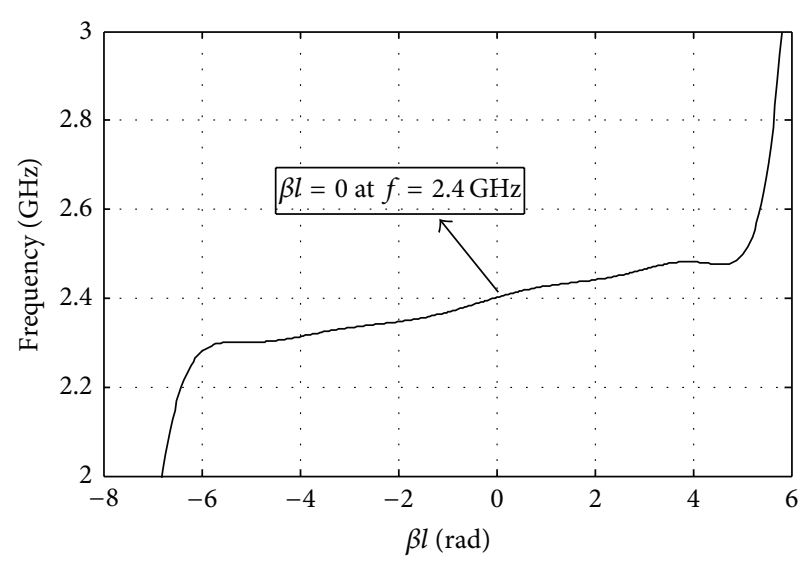

Figure 9: The dispersion response of the narrow-band filter.

ripples in the response curve is due to the CST simulation software, which is based on the time-domain analysis using a truncated Gaussian excitation signal. Consequently, the resulting bounded frequency band of excitation and the resonance nature of filter structure generate the ripples in the simulated response. The magnitude of ripples may be reduced by increasing the accuracy of simulations, but the time of computation greatly increases.

The frequency response of the single-band filter is shown in Figure 10. The graph covers a frequency range up to $10 \mathrm{GHz}$ to show that spurious response suppression is very good and is about $65 \mathrm{~dB}$.

The main advantage of the designed filter is its compactness and miniaturization. It is almost $70 \%$ smaller than those reported in the literature [7].

\section{Design of a Dual-Band Filter}

At first, a ZOR unit cell filter is designed for dual-band applications. The lower pass band is due to its left-handed resonance and the upper pass band is due to the right-handed 


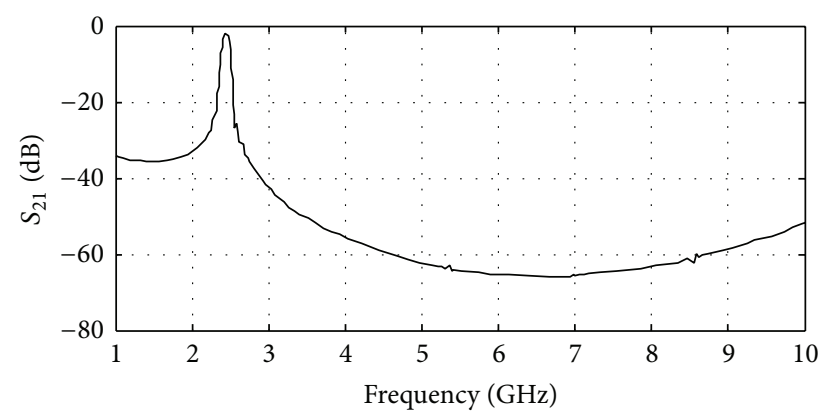

FIGURE 10: The spurious response of narrow-band filter as $S_{21}$ in the stop band up to $10 \mathrm{GHz}$.

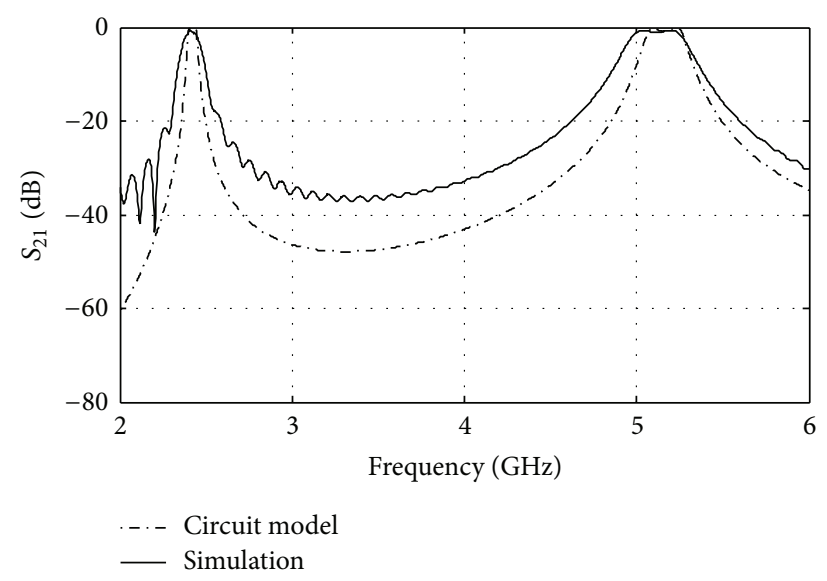

(a)

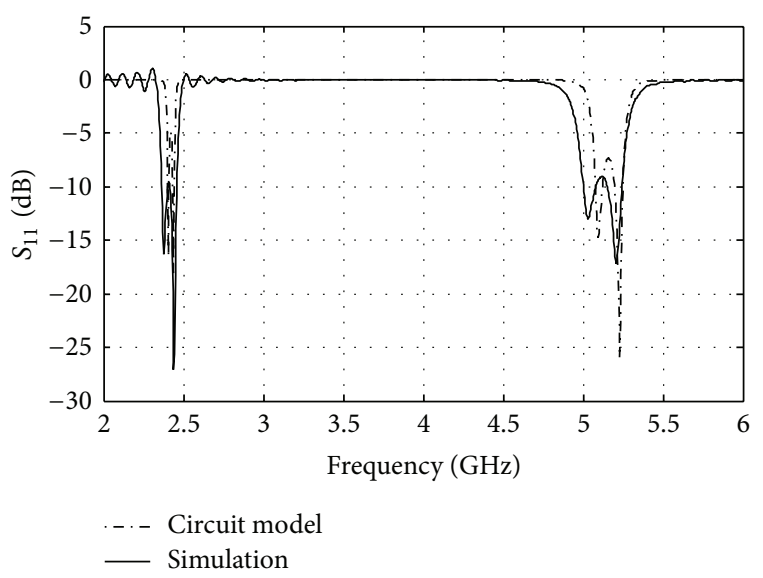

(b)

FIGURE 11: The frequency response of the dual-band filter as obtained by the circuit model and simulation. (a) $S_{21}$; (b) $S_{11}$.

resonance. Both upper and lower bands can be designed independently. The frequency response of the designed filter has achieved its specification. In order to improve the isolation between the two bands and also higher quality factor $(Q)$ at each band, two ZOR unit cells are connected again using inductive coupling. Its design specifications are as follows:

first band: center frequency, $f_{0}=2.44 \mathrm{GHz}$, bandwidth, $\mathrm{BW}=80 \mathrm{MHz}$, and maximum insertion loss, $\mathrm{IL}=0.5 \mathrm{~dB}$;

second band: center frequency, $f_{0}=5.2 \mathrm{GHz}$, bandwidth, $\mathrm{BW}=150 \mathrm{MHz}$, and maximum insertion loss, $\mathrm{IL}=0.5 \mathrm{~dB}$;

isolation between the two pass bands $=30 \mathrm{~dB}$.

For the construction of error function for the dual-band filter, the frequency intervals of each pass-band, each stop band, and each transition band are divided into 51, 212, and 45 discrete frequencies. The physical dimensions of each unit cell of the dual-band filter obtained for the optimum design are given in Table 3.

The frequency responses by circuit model and full wave computer simulation using CST Microwave Studio are illustrated in Figure 11. An isolation of $37 \mathrm{~dB}$ is observed between two pass bands in full wave simulation. The dispersion characteristic is extracted and shown in Figure 12.

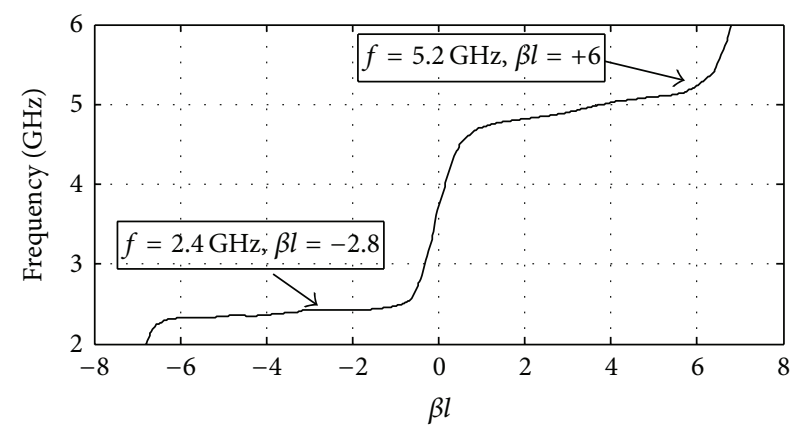

FIGURE 12: The dispersion curve of the dual-band filter.

The compactness of this filter is 64 percent better than the available designs reported in the literature $[2,15]$.

The difference between circuit model and full wave simulation results in Figures 8 and 11 is due to the fact that we used two different variables for gaps and widths of interdigital structures to provide more degree of freedom in full wave optimization.

To ensure maximum power transfer and reduction of return loss, a single open stub matching network is designed and added in parallel to the input of both filters to match source and load impedances. 
TABLE 3: Dimensions of dual-band filter designed by the proposed method.

(a) Dimensions of first unit cell

\begin{tabular}{|c|c|c|c|c|c|}
\hline \multicolumn{3}{|c|}{ Values before full wave optimization $(\mathrm{mm})$} & \multicolumn{3}{|c|}{ Values after full wave optimization (mm) } \\
\hline$w_{f}=0.23$ & $l_{s t}=2.00$ & $w_{t j 2}=0.10$ & $w_{f 1,2}=0.27 \& 0.20$ & $l_{s t}=1.10$ & $w_{t j 2}=0.10$ \\
\hline gap $=0.48$ & $w_{m}=0.24$ & $l_{m}=2.00$ & gap $=0.45$ & $w_{m}=0.10$ & $l_{m}=2.00$ \\
\hline$l_{f 1,2}=1.27 \& 1.26$ & $d_{1}=0.35$ & $d_{2}=0.37$ & $l_{f 1,2}=1.30 \& 1.70$ & $d_{1}=0.40$ & $d_{2}=0.40$ \\
\hline$w_{s t}=1.17$ & $w_{t j 1}=0.20$ & $d_{3}=4.03$ & $w_{s t}=1.12$ & $w_{t j 1}=0.20$ & $d_{3}=4.95$ \\
\hline
\end{tabular}

(b) Dimensions of second unit cell

\begin{tabular}{|c|c|c|c|c|c|}
\hline \multicolumn{3}{|c|}{ Values before full wave optimization (mm) } & \multicolumn{3}{|c|}{ Values after full wave optimization (mm) } \\
\hline$w_{f}=0.3$ & $l_{s t}=1.90$ & $w_{t j 2}=0.10$ & $w_{f}=0.25$ & $l_{s t}=1.20$ & $w_{t j 2}=0.10$ \\
\hline gap $=0.47$ & $w_{m}=0.24$ & $l_{m}=2.00$ & gap $=0.40$ & $w_{m}=0.10$ & $l_{m}=2.00$ \\
\hline$l_{f 1,2}=1.40 \& 1.30$ & $d_{1}=0.50$ & $d_{2}=0.50$ & $l_{f 1,2}=1.10 \& 1.30$ & $d_{1}=0.50$ & $d_{2}=0.50$ \\
\hline$w_{s t}=1.10$ & $w_{t j 1}=0.20$ & $d_{3}=4.30$ & $w_{s t}=1.14$ & $w_{t j 1}=0.20$ & $d_{3}=4.95$ \\
\hline
\end{tabular}
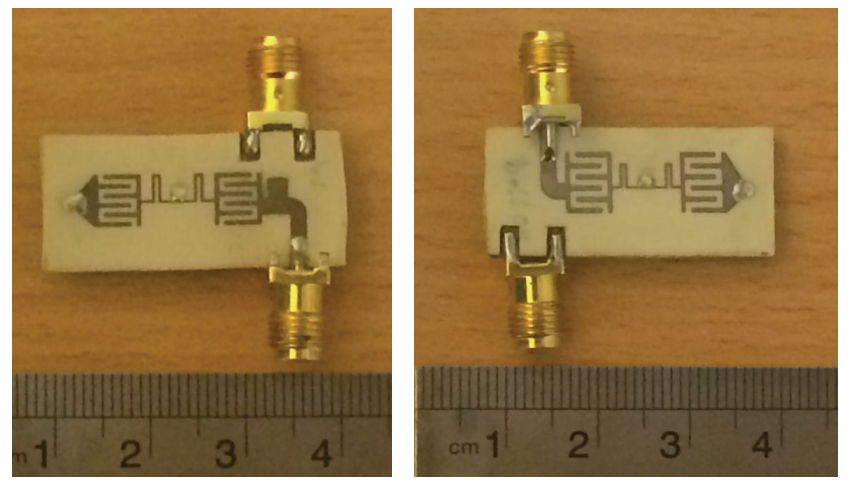

(a)
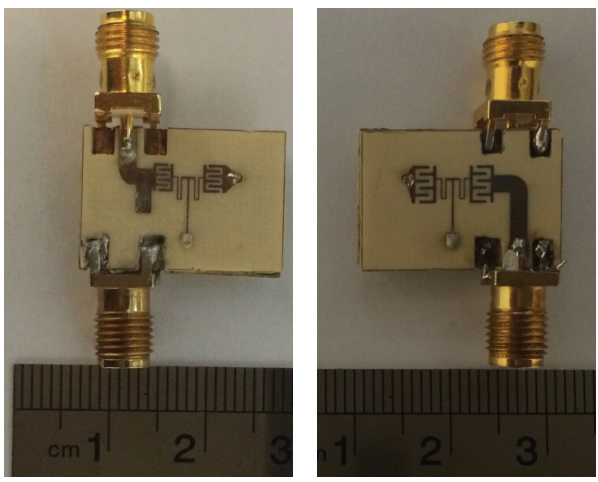

(b)

FIGURE 13: Photographs of the fabricated prototype models. (a) Narrow-band filter; (b) dual-band filter.

\section{Measurement Result}

The prototypes of the fabricated narrow-band and dual-band band pass filters are depicted in Figure 13. Substrate Rogers 4003 is used with dielectric constant 3.55, thickness 20 mil, and $\tan \delta=0.0027$. For the miniaturization of filter profiles, the two unit cells of filters are located together back to back by a pin, so that the ground plane is placed between them. The dimensions of the pin are optimized in the full wave simulation. The frequency responses of the narrow-band and dual-band filters as obtained by full wave simulation and measurement are illustrated in Figures 14 and 15, for $S_{21}$ versus frequency.

The agreement among simulation and measurement is fairly good. The difference between the simulation results and measurement data is due to the mediocre fabrication techniques and low precision of the equipment in our laboratory.

\section{Conclusion}

The zero order resonators derived from the theory of metamaterials are used as the building blocks to design narrowband and dual-band filters. A circuit model is derived for

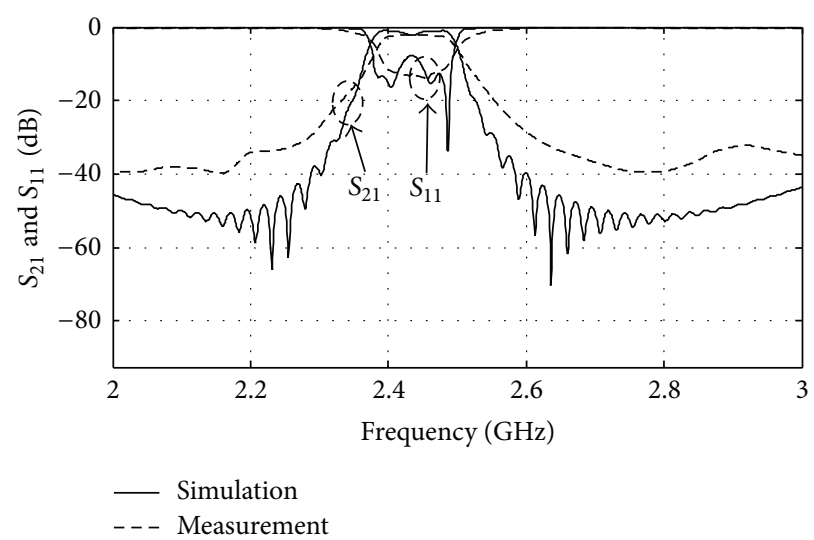

FIGURE 14: Results for simulation and measurement of the narrowband filter (the dimensions of substrate are $1.4 \times 3.2 \mathrm{~mm}^{2}$ ).

the filters by the method of least squares. The filter designs are for single- and dual-band application in WLAN systems. The proposed designs have achieved $70 \%$ and $64 \%$ miniaturization for the narrow-band and dual-band filters compared to the available models in the literature. The measurement and computer simulation data of fabricated prototypes agree quite 


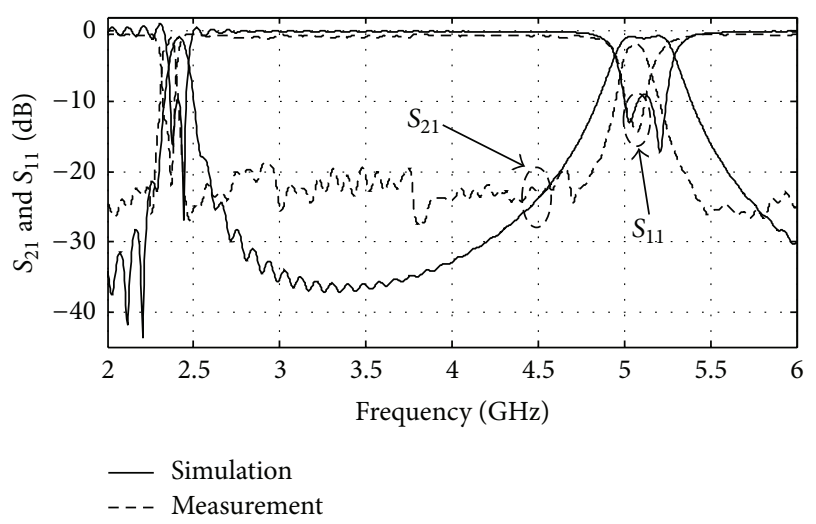

FIGURE 15: Results for simulation and measurement of $S_{21}$ for the dual-band filter (the dimensions of substrate are $2 \times 1.4 \mathrm{~mm}^{2}$ ).

well with the results of the proposed equivalent circuits of the filter. Consequently, the proposed filter structures and design method are verified for application in microwave systems.

\section{Conflict of Interests}

The authors declare that there is no conflict of interests regarding the publication of this paper.

\section{References}

[1] J.-T. Kuo and H.-S. Cheng, "Design of quasi-elliptic function filters with a dual-passband response," IEEE Microwave and Wireless Components Letters, vol. 14, no. 10, pp. 472-474, 2004.

[2] S. Sun and L. Zhu, "Coupling dispersion of parallel-coupled microstrip lines for dual-band filters with controllable fractional pass bandwidths," in Proceedings of the IEEE MTT-S International Microwave Symposium, p. 4, IEEE, June 2005.

[3] C. Caloz and T. Itoh, Electromagnetic Metamaterials: Transmission Line Theory and Microwave Applications, John Wiley \& Sons, 2005.

[4] S. Kahng, G. Jang, B. Lee, J. Ju, and S. Lee, "Compact UHF bandpass filter with the subwavelength metamaterial ZORs and transmission zeros for enhanced channel selectivity," in Proceedings of the 41st European Microwave Conference (EuMC '11), pp. 567-570, IEEE, October 2011.

[5] G. Jang and S. Kahng, "Design of a dual-band metamaterial band-pass filter using zeroth order resonance," Progress In Electromagnetics Research C, vol. 12, pp. 149-162, 2010.

[6] C. H. Tseng and T. Itoh, "Dual-band bandpass and bandstop filters using composite right/left-handed metamaterial transmission lines," in Proceedings of the IEEE MTT-S International Microwave Symposium Digest, pp. 931-934, IEEE, June 2006.

[7] X. Chen, J. Li, and Y. Shen, "WLAN bandpass filter with wide stopband using spurlines and microstrip stubs," in Proceedings of the 9th International Symposium on Antennas Propagation and EM Theory (ISAPE '10), pp. 1188-1191, December 2010.

[8] T. C. Edwards, Foundations for Microstrip Circuit Design, Wiley, Chichester, UK, 1981.

[9] G. D. Alley, "Interdigital capacitors and their application to lumped-element microwave integrated circuits," IEEE Transactions on Microwave Theory and Techniques, vol. 18, no. 12, pp. 1028-1033, 1970.
[10] R. Siragusa, H. V. Nguyen, P. Lemaître-Auger, S. Tedjini, and C. Caloz, "Modeling and synthesis of the interdigital/stub composite right/left-handed artificial transmission line," International Journal of RF and Microwave Computer-Aided Engineering, vol. 19, no. 5, pp. 549-560, 2009.

[11] B. C. Wadell, Transmission Line Design Handbook, Artech House, 1991.

[12] D. M. Pozar, Microwave Engineering, John Wiley \& Sons, 2009.

[13] H. Oraizi and N. Azadi-Tinat, "Optimum design of novel UWB multilayer microstrip hairpin filters with harmonic suppression and impedance matching," International Journal of Antennas and Propagation, vol. 2012, Article ID 762790, 7 pages, 2012.

[14] S.-G. Mao, S.-L. Chen, and C.-W. Huang, "Effective electromagnetic parameters of novel distributed left-handed microstrip lines," IEEE Transactions on Microwave Theory and Techniques, vol. 53, no. 4, pp. 1515-1521, 2005.

[15] J. T. Kuo and H. S. Cheng, "Design of quasi-elliptic function filters with a dual-passband response," IEEE Microwave and Wireless Components Letters, vol. 14, no. 10, pp. 472-474, 2004. 

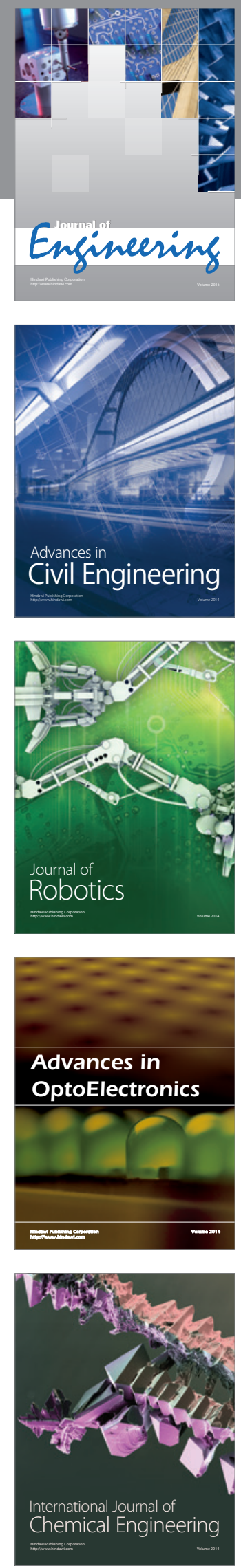

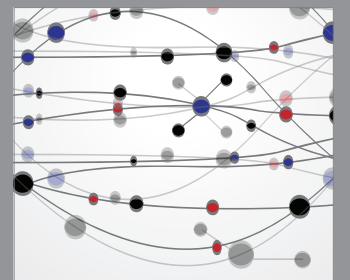

The Scientific World Journal
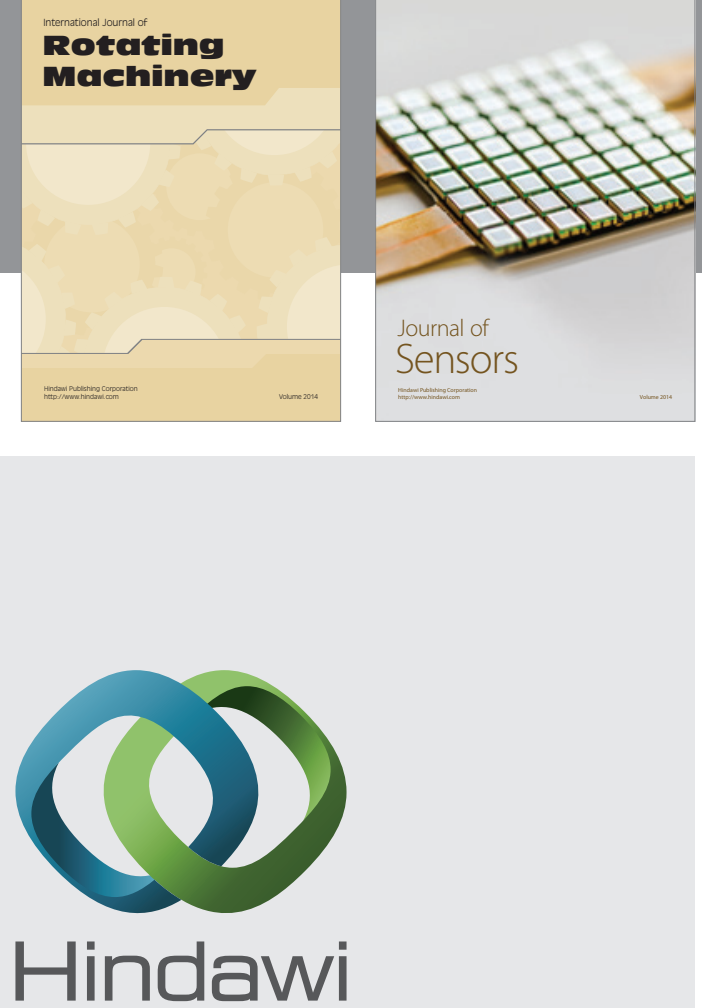

Submit your manuscripts at http://www.hindawi.com
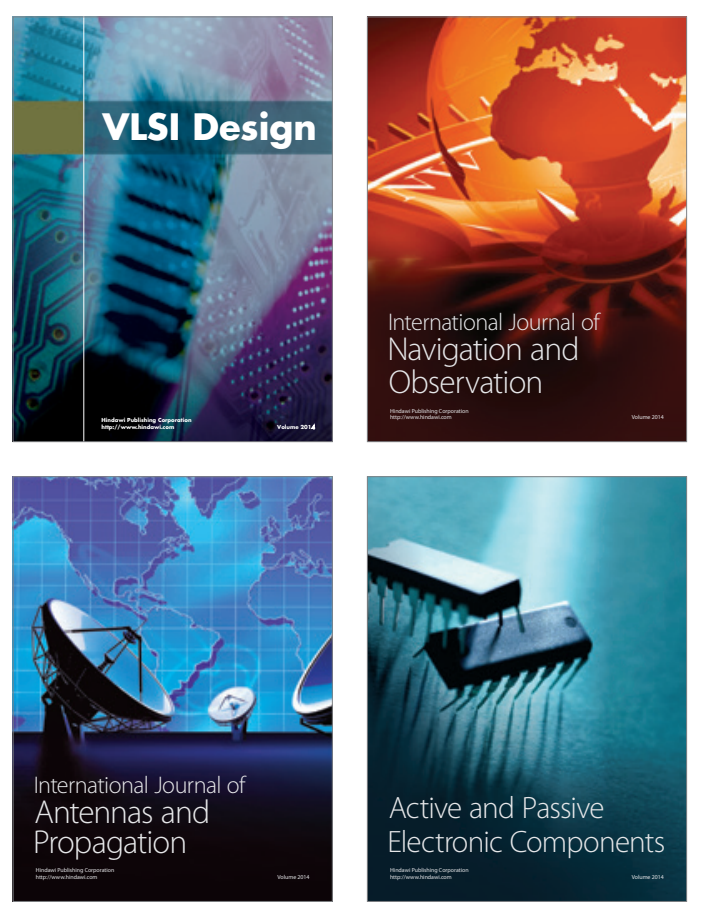
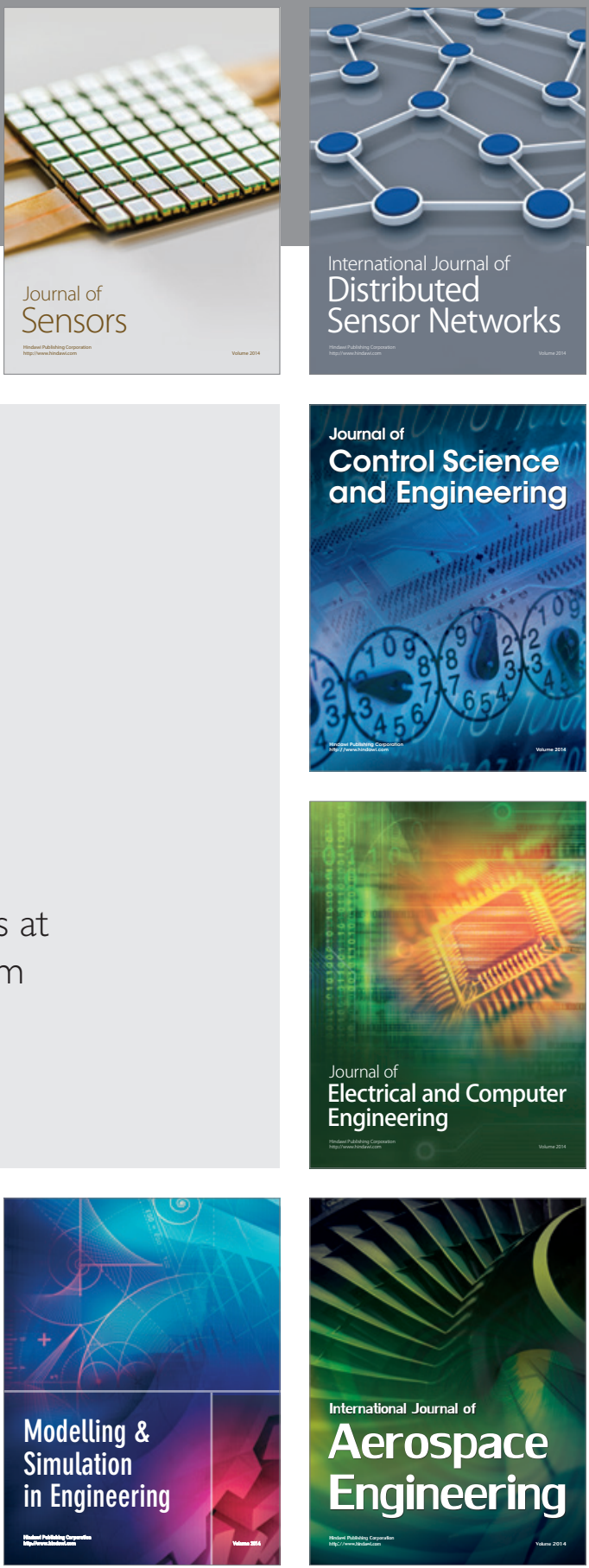

Journal of

Control Science

and Engineering
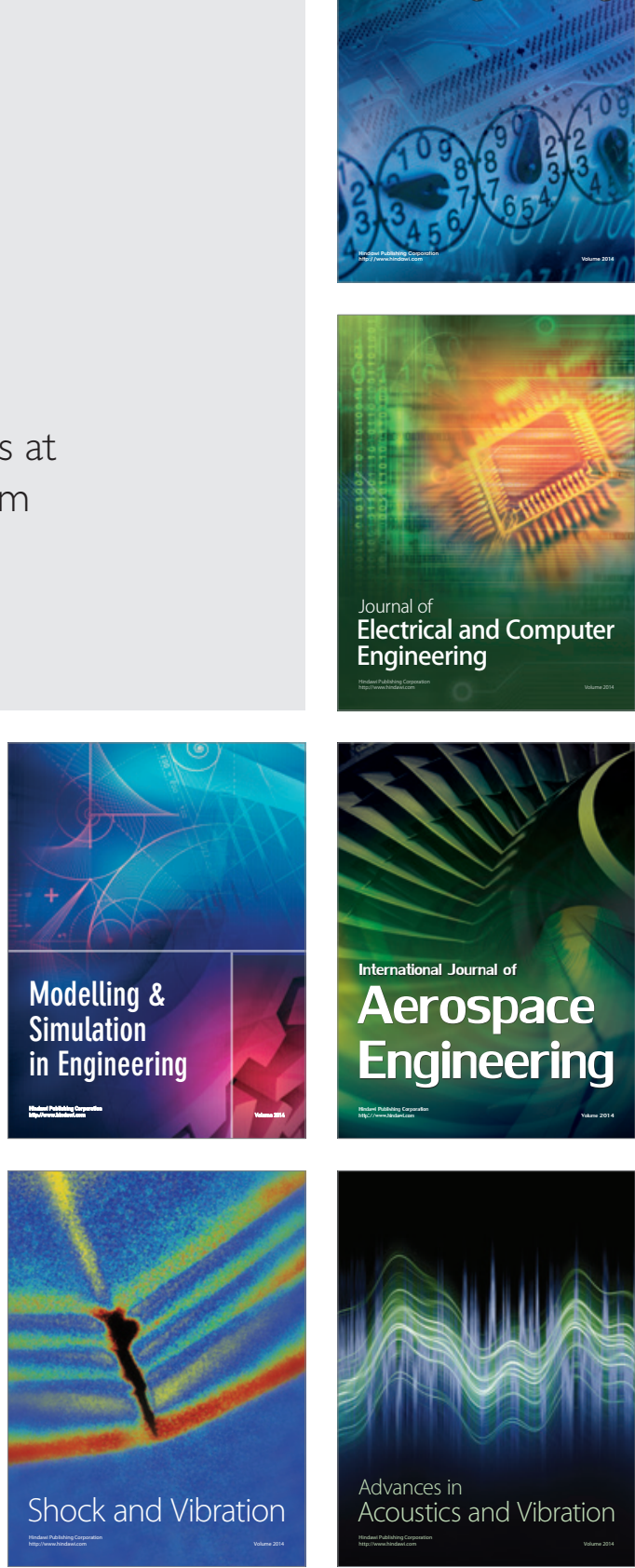